\title{
A Variable Structure-Based Estimation Strategy Applied to an RRR Robot System
}

\author{
Jacob Goodman, Jinho Kim \\ Department of Mechanical Engineering, University of Maryland, Baltimore County (UMBC), \\ 1000 Hilltop Circle, Baltimore, Maryland, USA, 21250 \\ Andrew S. Lee, S. Andrew Gadsden \\ School of Engineering, University of Guelph, \\ Guelph, Ontario, Canada, N1G 2 WI \\ Mohammad Al-Shabi \\ Department of Mechanical Engineering, University of Sharjah \\ Sharjah, UAE
}

E-mail:.jg4@umbc.edu,mbcjhkim@umbc.edu,alee32@uoguelph.ca,gadsden@uoguelph.ca,malshabi@sharjah.ac.ae

\begin{abstract}
Nonlinear estimation strategies are important for accurate and reliable control of robotic manipulators. This brief paper studies the application of estimation theory to a simple robotic manipulator. Two estimation techniques are considered: the classic extended Kalman filter (EKF), and the robust smooth variable structure filter (SVSF). The EKF is included to present a basic background in estimation techniques and the SVSF is described and implemented on the system. We simulate the SVSF applied to a dynamically modeled three-link robotic manipulator. The results of the paper demonstrate that nonlinear estimation techniques such as the SVSF can be applied effectively to robots with modeling uncertainty and external disturbances.
\end{abstract}

Keywords: Robotic manipulator, estimation theory, Kalman filter, smooth variable structure filter.

\section{Introduction}

The accurate and predictable control of mechanical and electrical systems is dependent on the precise knowledge of system states and parameters. However, accurate knowledge of these states is not usually available, and instead, some form of estimate is calculated. Estimates are usually based on available measurement data as well as knowledge of the system model and history. Estimation theory concerns itself with the various techniques with which accurate and robust estimates can be made. Some estimators are referred to as 'filters' since they extract true signals from noisy measurements.
Many estimation strategies are well-suited for linear systems in the discrete time domain. Systems can be generalized into four main categories (with physical systems only existing in the latter three): (i) linear; (ii) approximately linear systems; (iii) medium to strongly non-linear systems; and (iv) systems for whom nonlinearity is the main characteristic ${ }^{1}$. For categories (i) and (ii), typical estimations strategies such as the Kalman filter (KF) work well. For category (iii), estimation strategies can be extended with the use of linearization techniques such as a Jacobian or first-order Taylor series. Category (iv) systems can present a significant challenge for any estimation or control system. 
Robotic manipulators have become ubiquitous over the last several decades. Many significant advances in manufacturing and automation are due to effective implementation of robotic manipulators. Robotic manipulators have achieved great utility in their ability to perform repetitive tasks in a highly consistent manner. As such, estimation techniques play a critical role in the performance of these systems.

In this brief paper, we shall consider a relatively simple planar triple-rotary (RRR) robotic manipulator. The dynamics of even such a simple system are quite nonlinear. For context, we provide a basic overview of the classic estimation algorithm known as the Kalman filter (KF), and its nonlinear version known as the extended Kalman filter (EKF). We then present a robust estimation strategy referred to as the smooth variable structure filter (SVSF). We demonstrate the application of the SVSF to a simple RRR manipulator and summarize the results.

The paper is organized as follows: Section 2 presents a brief background of the EKF and SFVF; Section 3 provides the system dynamics and basic control loop; the simulation results are shown in Section 4; and, Section 5 summarizes the paper and provides suggestions for future work.

\section{Kalman \& Smooth Variable Structure Filters}

\section{The Kalman Filter}

For a linear system, state dynamics can be expressed in state representation form as follows ${ }^{1}$ :

$$
\begin{gathered}
x_{k+1}=A x_{k}+B u_{k}+w_{k} \\
z_{k+1}=C x_{k+1}+v_{k+1}
\end{gathered}
$$

In (1), $x_{k}$ refers to the system states. $A$ is the linear system matrix, $B$ is the input gain matrix, $u_{k}$ is the input vector and $w_{k}$ is the system noise. In (2) $z_{k}$ is the measurement vector, $C$ is the linear measurement matrix, and $v_{k}$ represents the measurement noise.

The Kalman filter assumes that the system model is well known and linear, the initial states are known, and the measurement and system noise is Gaussian with zero mean and known respective covariance matrices. ${ }^{1,2}$ The KF works as a predictor-corrector; the system model is used to obtain an a priori estimate of the states, whereupon measurements combined with the Kalman Gain matrix are used to apply a correction term to create an updated a posteriori state estimate. Figure 2 illustrates the general concept.

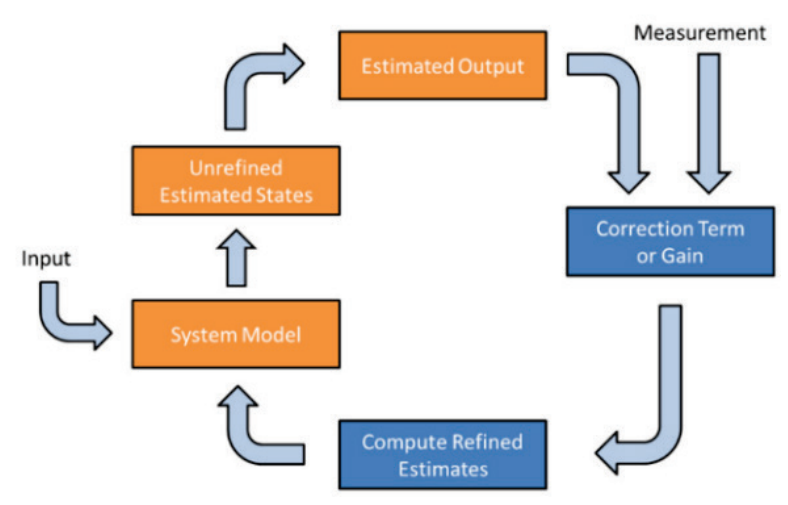

Figure 1. Predictor Estimator ${ }^{1}$

For a nonlinear system, equation's (1) and (2) become:

$$
\begin{aligned}
& x_{k+1}=f\left(x_{k}, u_{k}\right)+w_{k} \\
& z_{k+1}=h\left(x_{k+1}\right)+v_{k+1}
\end{aligned}
$$

where $f$ and $h$ are the nonlinear system and measurement models respectively.

The EKF follows the basic procedure of the KF. When computing the predicted and updated state error covariance matrices, where the nonlinear equations cannot be used, the strategy of the EKF is to linearize $f$ and $h$ around the current state estimate by means of the Jacobian. While this algorithm is effective, it does come at the price of optimality as well as robustness. Linearization can fail to account for some modeling uncertainties which can lead to instability ${ }^{1}$. More in depth treatment of the Kalman filter can be found in the literature. ${ }^{3,4,5}$

\section{The Smooth Variable Structure Filter}

The basic form of the smooth variable structure filter (SVSF) first appeared in $2007 .^{2}$ Formulated as a predictor-corrector, it is based on variable structure theory as well as sliding mode concepts. ${ }^{1,2}$. The basic idea is to use a switching gain to push estimates to within a defined boundary of the true states; termed the existence subspace. ${ }^{1}$ Important prerequisites of the SVSF is that the system in question be differentiable as well as observable. ${ }^{6}$ An in-depth treatment of the concepts can be found in the reference literature. ${ }^{1,2,6-20}$ The basic SVSF concept is illustrated in Figure 2. 


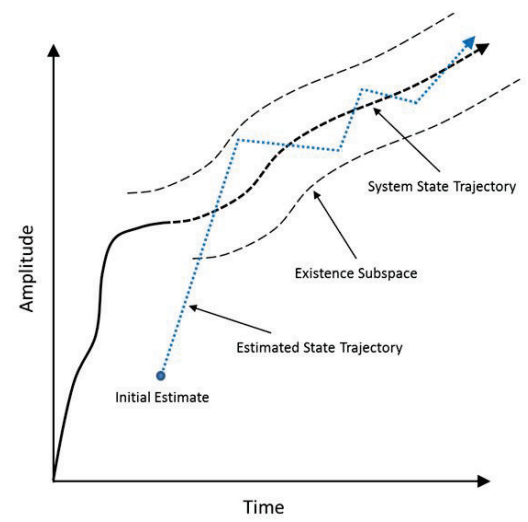

Figure 2. SVSF Concept Diagram ${ }^{2}$

\section{RRR Manipulator Dynamics}

The basic configuration of an RRR robotic manipulator appears in Figure 3 below. Note the important parameters include the rod masses $m_{i}$, center of gravity lengths $l_{i}$, and overall lengths $L_{i}$. From these the moments of inertias $J_{i}$ can be computed. $\theta_{i}$ are the joint variables. For all parameters $i=1,2,3$.

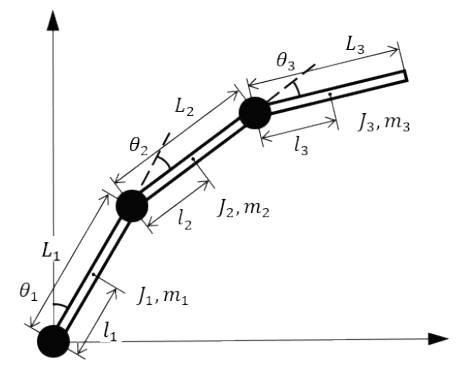

Figure 3. RRR Planer Manipulator

The generalized equation for a frictionless robotic arm can shown to be ${ }^{7}$ :

$$
M(\theta) \ddot{\theta}+B(\dot{\theta}, \theta) \dot{\theta}+G(\theta)=u
$$

where $\theta$ refers to the joint variable matrix with $n$ degrees of freedom. In the case of an RRR manipulator $n=3 . M$ is the inertia matrix, $V$ is the Coriolis vector, $G$ is the gravity vector, and $u$ is the control input vector. Refer to the following reference for additional details. ${ }^{7}$

\section{Simulation Results and Brief Discussion}

For simulation, consider a simplified RRR manipulator with unitary masses and moments of inertia. Reference inputs are simple sinusoids. A simple PD controller is used to drive the system inputs. ${ }^{7}$ Both an EKF and SVSF were applied to the measurement feedback loop for improved estimates of the joint angles.
Figures 4 and 5 show the angle and velocity inputs and the corresponding system responses. Note that the SVSF was able to yield very good estimates of the joint angles which can be fed back into a nonlinear controller for better system response and control. In terms of trajectory following accuracy, the SVSF offered an improvement of over $30 \%$ when compared to the EKF.

The performance of the EKF is reduced due to the approximations of the nonlinearities by use of a firstorder Taylor series expansion. The SVSF does not require any sort of linearization such that the true state trajectory can be more closely approximated. The SVSF offers a robust estimation strategy whereby the switching function included in the gain maintains the estimates close to the true state trajectory.

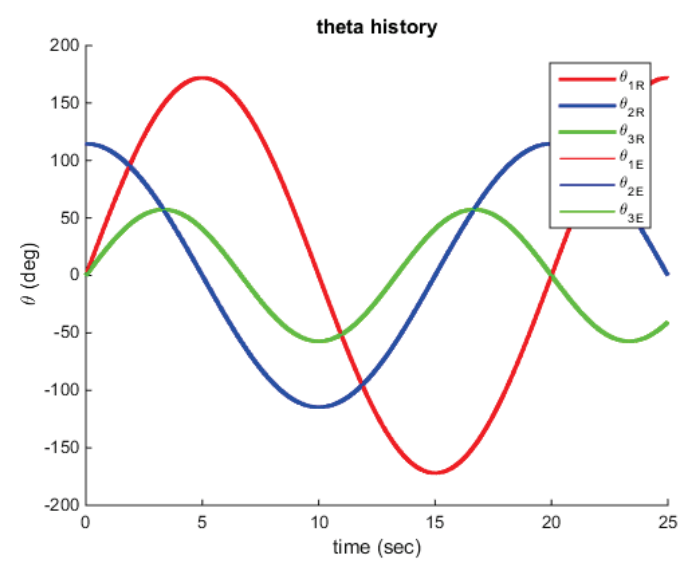

Figure 4. Angle Input and Response

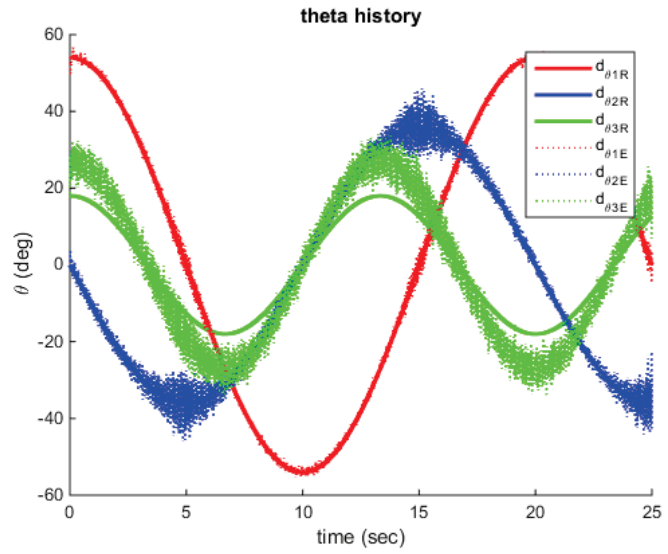

Figure 5. Velocity Input and Response 


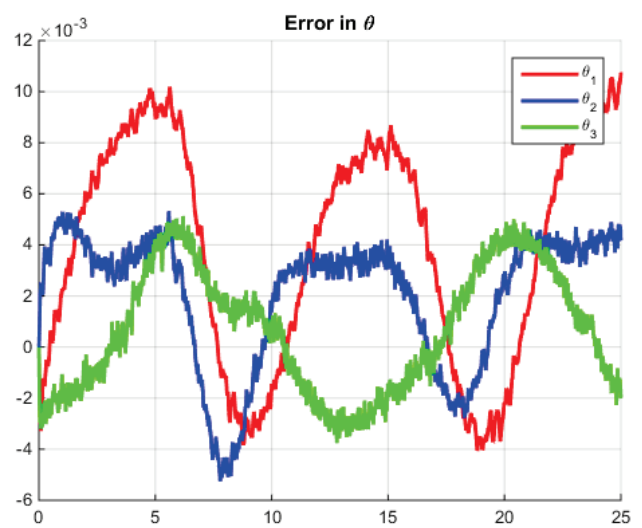

Figure 6. Angle Errors

\section{Concl usions and Future Work}

Estimation theory has profound utility to a wide-variety of control and general engineering problems. Robotic manipulators, in their requirement for precise and consistent control, represent an important application of accurate and robust estimation techniques. Filters such as the SVSF are particularly suited for robotic manipulators as manipulator dynamics are by necessity almost always nonlinear. The results demonstrate that the SVSF can be effectively applied to an idealized robotic arm. For future work: more realistic model conditions will be considered, and the SVSF, as well as other estimation strategies, can be assessed for both robustness and accuracy.

\section{References}

1. J. R. Leigh, Control Theory, 2nd Ed. The Institution of Electrical Engineers, London, 2004.

2. S. A. Gadsden, Smooth Variable Structure Filtering: Theory and Applications, Department of Mechanical Engineering, McMaster University, Ph.D Thesis, Hamilton, Ontario, 2011.

3. R. E. Kalman, A New Approach to Linear Filtering and Prediction Problems, Journal of Basic Engineering, Transactions of ASME, 82, 1960, 35-45.

4. B. D. O. Anderson and J. B. Moore, Optimal Filtering, Prentice-Hall Englewood, NJ, 1979.

5. A. Gelb, Applied Optimal Estimation, Cambridge, MA: MIT Press, 1974.
6. M. Al-Shabi, The General Toeplitz/Observability SVSF, Department of Mechanical Engineering, McMaster University, Hamilton, Ontario, 2011.

7. F. L. Lewis, D. M. Dawson and C. T. Abdallah, Robot Manipulator Control: Theory and Practice, 2nd Ed., Marcel Dekker Inc., New York, 2004.

8. S. A. Gadsden and S. R. Habibi, A New Robust Filtering Strategy for Linear Systems, ASME Journal of Dynamic Systems, Measurements, and Control, 135, 2013.

9. S. A. Gadsden, Y Song, and S. R. Habibi, Novel ModelBased Estimators for the Purposes of Fault Detection and Diagnosis, IEEE/ASME Transactions on Mechatronics, 18, 2013.

10. S. A. Gadsden, S. R. Habibi, and T. Kirubarajan, Kalman and Smooth Variable Structure Filters for Robust Estimation, IEEE Transactions on Aerospace and Electronic Systems, 50, 2014.

11. S. A. Gadsden, M. Al-Shabi, and S. R. Habibi, Estimation Strategies for the Condition Monitoring of a Battery System in a Hybrid Electric Vehicle, ISRN Signal Processing, 2011.

12. S. A. Gadsden, S. R. Habibi, and T. Kirubarajan, The Smooth Particle Variable Structure Filter, Transactions of the Canadian Scoiety for Mechanical Engineering, 36(2), 2012, 177-193.

13. S. A. Gadsden, D. Dunne, S. R. Habibi, and T. Kirubarajan, Nonlinear Estimation Techniques Applied on Target Tracking Problems, ASME Journal of Dynamic Systems, Measurement, and Control, 134(5), 2012.

14. M. Al-Shabi, S. A. Gadsden, and S. R. Habibi, Kalman Filtering Strategies Utilizing the Chattering Effects of the Smooth Variable Structure Filter, Signal Processing, 93(2), 2013, 420-431.

15. H. H. Afshari, S. A. Gadsden, and S. R. Habibi, Gaussian Filters for Parameter and State Estimtaion: A General Review and Recent Trends, Signal Processing, 135, 2017, 218-238.

16. S. A. Gadsden and A. S. Lee, Advances of the Smooth Variable Structure Filter: Square-Root and Two-Pass Formulations, Journal of Applied Remote Sensing, 11(1), 2017.

17. J. Goodman, J. Kim, A. S. Lee, S. A. Gadsden, and M. AlShabi, Nonlinear Estimation Strategies Applied on an RRR Robotic Manipulator, The 2017 International Conference on Artificial Life and Robotics (ICAROB2017), Miyazaki, Japan, 2017.

18. J. Kim, A. S. Lee, K. Chang, B. Schwarz, S. A. Gadsden, and M. Al-Shabi, Dynamic Modeling and Motion Control of a Three-Link Robotic Manipulator, The 2017 International Conference on Artificial Life and Robotics (ICAROB2017), Miyazaki, Japan, 2017 